\title{
The role of ramucirumab with docetaxel in epidermal growth factor receptor mutant and wild-type non-small cell lung cancer
}

\author{
Tim Ellis-Caleo ${ }^{1}$, Joel W. Neal ${ }^{2}$ \\ ${ }^{1}$ Department of Medicine, Stanford University School of Medicine, Stanford, CA, USA; ${ }^{2}$ Division of Oncology, Department of Medicine, Stanford \\ Cancer Institute, Stanford University School of Medicine, Stanford, CA, USA \\ Contributions: (I) Conception and design: T Ellis-Caleo, JW Neal; (II) Administrative support: T Ellis-Caleo, JW Neal; (III) Provision of study \\ materials or patients: T Ellis-Caleo, JW Neal; (IV) Collection and assembly of data: T Ellis-Caleo; (V) Data analysis and interpretation: T Ellis- \\ Caleo, JW Neal; (VI) Manuscript writing: Both authors; (VII) Final approval of manuscript: Both authors. \\ Correspondence to: Joel W. Neal, MD, PhD. Department of Medicine, Division of Oncology, Stanford Cancer Institute, Stanford University School of \\ Medicine, 875 Blake Wilbur Drive, Stanford, CA 94305, USA. Email: jwneal@stanford.edu.
}

Background: Ramucirumab paired with docetaxel extends progression free survival and overall survival in non-small cell lung cancer (NSCLC) following progression on platinum therapy. There is some data that epidermal growth factor receptor (EGFR) mutant disease would respond better to vascular endothelial growth factor receptor (VEGFR) therapy than EGFR wild type disease.

Methods: This retrospective, single-institution cohort study reports outcomes of patients who received docetaxel with or without ramucirumab according to EGFR status. Clinical data including age, performance status, metastatic burden and prior treatment history was obtained and reported with time on treatment and overall survival as primary endpoints. Data analysis was performed for three cohorts: EGFR mutant disease receiving docetaxel and ramucirumab (EGFR-doce/ram), EGFR mutant disease receiving docetaxel alone (EGFR-doce) and EGFR wild type disease receiving docetaxel and ramucirumab (WT-doce/ram).

Results: Patients in the EGFR-doce/ram cohort had a median time on docetaxel of 1.4 months (95\% CI: 0.72-5.2 months) and of 0.8 months (95\% CI: 0.2-6.5 months) on ramucirumab. Patients in the EGFR-doce cohort were on docetaxel for a median 1.4 months (95\% CI: 0.9-2.4 months). Patients in the WT-doce/ram cohort had a median time on docetaxel of 2.3 months (95\% CI: 1.6-4.1 months) and on ramucirumab of 1.4 months (95\% CI: 0.8-3.2 months). There was no significant difference between time on ramucirumab or docetaxel between the cohorts. Overall survival for the three cohorts was noted to be 6.7 months $(95 \%$ CI: 2.5-16.2 months) for the EGFR-doce/ram cohort, 4.9 months (95\% CI: 4.2-12.5 months) for the EGFR-doce cohort and 6.6 months (95\% CI: 4.3-12.8 months) for the WT-doce/ram cohort. There was no significant difference in overall survival between the cohorts.

Conclusions: Our data did not support the initial hypothesis that patients with EGFR mutant disease would do better with the addition of ramucirumab. Our study was limited by small sample size, retrospective nature and inability to control for confounders including prior bevacizumab or immune checkpoint inhibitor (ICI) exposure. This study offers real-world estimates to clinicians and patients about the length of time they can expect to derive benefit from the combination of ramucirumab and docetaxel.

Keywords: Non-small cell lung cancer (NSCLC); ramucirumab; docetaxel; epidermal growth factor receptor (EGFR)

Submitted Mar 03, 2021. Accepted for publication Jul 09, 2021.

doi: $10.21037 /$ jtd-21-557

View this article at: https://dx.doi.org/10.21037/jtd-21-557

(C) Journal of Thoracic Disease. All rights reserved. 


\section{Introduction}

Before 2014, second line therapy options for advanced nonsmall cell lung cancer (NSCLC) following progression on platinum-based chemotherapy were docetaxel or pemetrexed, with consideration of other single agent drugs such as gemcitabine (1). Reported in 2014, the REVEL study tested the addition of ramucirumab, an anti-vascular endothelial growth factor receptor (VEGFR) monoclonal antibody to docetaxel for patients with NSCLC who had progressed through platinum-based therapy (2). Ramucirumab was shown to extend both progression free survival (4.5 vs. 3.0 months) and overall survival (10.5 vs. 9.1 months) by approximately one and a half months. These improvements to PFS and OS came with increases in toxicity - most notably fatigue, cytopenias and hypertension. These findings established ramucirumab as an option in combination with second line chemotherapy for NSCLC with a good ECOG performance status [0-1]. However, adoption into clinical practice was slow, likely in part due to the concurrent emergence of immune checkpoint inhibitor (ICI) therapy as another second line option in early 2015. As platinum chemotherapy with the addition of ICI therapy has become a new first-line standard, generally without anti-VEGF(R) therapy, the combination of docetaxel with ramucirumab as second line therapy has become more compelling.

However, questions remain regarding the subpopulations which may benefit the most from this treatment. Most notably, tumors with epidermal growth factor receptor (EGFR) mutations account for about $15 \%$ of non-small cell lung adenocarcinoma in the United States and are exquisitely sensitive to first line treatment with small EGFR tyrosine kinase inhibitors (TKIs), leaving chemotherapy options for later lines of therapy. In the REVEL study, both EGFR mutant and EGFR wild type patients were included, although the EGFR status was unknown for a majority of patients.

There is a paucity of biomarkers to predict response to anti-VEGF(R) therapy; however, EGFR mutant disease has stood out as the best predictor of response known currently. Significant pre-clinical data supports the synergistic effect of inhibition of both EGFR and VEGF pathways (3). Clinically, ramucirumab has shown to be effective in the frontline setting when added to TKI therapy in EGFR mutant NSCLC. In the RELAY study, ramucirumab increased progression free survival (19.4 vs. 12.4 months) when added to erlotinib in the front line setting for EGFR mutant disease (4). Ramucirumab is now an FDA approved therapy, but adoption has been slow in the new era of first-line treatment with the EGFR inhibitor osimertinib. Progression free survival was also extended in EGFR mutated NSCLC by the addition of bevacizumab, another inhibitor of the VEGF pathway, to erlotinib in the front line setting in the interim analysis of NEJ026 (5). However, neither of these studies have demonstrated a significant benefit in overall survival with the combination strategy. A 2016 study of the addition of ramucirumab to docetaxel for second line therapy in Japanese patients included a subgroup of EGFR mutant patients who had received TKI therapy before or after platinum based therapy; however, it did not have sufficient numbers to show any statistically significant trends (6). Therefore, it is currently unclear how to optimally incorporate anti- $\operatorname{VEGF}(\mathrm{R})$ therapies in the treatment of EGFR mutant NSCLC.

Further clarity in the real-world performance of ramucirumab as part of second line therapy, generally after prior platinum-based chemotherapy and the option of ICI therapy, may help clinicians judge which patients would receive the greatest benefit, whether those with EGFR mutations or best performance status. This single institution retrospective study characterizes the real-world performance of ramucirumab when added to docetaxel in EGFR mutant disease as measured by time on therapy, as compared with patients without EGFR mutant lung cancer on docetaxel and ramucirumab. Our hypothesis was that patients with EGFR mutant lung cancer may have better outcomes on the combination therapy than patients in either of the other two groups. We describe and analyze outcomes among three cohorts of patients-those with EGFR mutations who received docetaxel and ramucirumab (EGFR-doce/ram), those with EGFR mutations who received only docetaxel (EGFR-doce) and those without EGFR mutations who received docetaxel and ramucirumab (WT-doce/ram).

We present the following article in accordance with the STROBE reporting checklist (available at https://dx.doi. org/10.21037/jtd-21-557).

\section{Methods}

\section{Patient selection and data extraction}

In this retrospective, single institution cohort study, we used the electronic health record based institutional outcomes database for clinical research (STARR) and an 
IRB approved protocol to find patients with NSCLC who had received docetaxel with or without ramucirumab. Patients were included if they had received treatment at Stanford Health Care, were diagnosed with biopsy confirmed stage IV NSCLC of either squamous or nonsquamous histology and received either docetaxel or docetaxel and ramucirumab regardless of prior treatment history. Patients enrolled started treatment between 2009 and 2020. Patients were excluded if EGFR mutation status was unknown or if they had a second synchronous malignancy. History of biopsy confirmed NSCLC was ascertained by CPT code search through STARR with manual verification of biopsy reports by the study team. Exposure to docetaxel or ramucirumab was performed by query of pharmacy administration records. EGFR status was ascertained by manual review of biopsy reports. ECOG performance status was obtained via clinician note immediately prior to therapy initiation. The presence of brain and liver metastases was obtained via manual review of staging studies immediately prior to the initiation of therapy. If the imaging studies were not directly available in STARR but the presence of these metastases were clearly noted in the treating oncologist's notes, they were recorded in our study. Clinician notes were used to determine prior exposure to bevacizumab, TKIs, ICIs and total prior lines of therapy. Missing data was noted in the reported tables. The study was conducted in accordance with the Declaration of Helsinki (as revised in 2013). The study was approved by institutional ethics board of Stanford University (IRB \#21319) and individual consent for this retrospective analysis was waived.

\section{Outcomes}

Our primary endpoint of time on therapy was defined as the time between the first and last administration of docetaxel and first and last administration of ramucirumab. If patients received only a single dose of therapy, they were recorded as having zero days on therapy. Overall survival was obtained via direct chart review and defined as the time from first dose of treatment regimen to death. Data for time on therapy and mortality was collected through July 30th, 2020. Censoring was performed for patients still receiving therapy and still alive as of the end of data collection. A single patient in the EGFR-doce cohort was lost to follow up immediately after the first dose of therapy and was excluded from the time on treatment and survival analyses.

\section{Statistical analysis}

Data was analyzed according to the three cohorts previously described-EGFR-doce/ram, EGFR-doce and WT-doce/ ram. We compared time on treatment and overall survival between the EGFR-doce/ram and EGFR-doce to isolate the effect of ramucirumab in EGFR mutated patients. The same analysis was carried out to compare the EGFR-doce/ ram cohort with the WT-doce/ram cohort to examine the effect of EGFR mutated disease on the response to the combination of docetaxel and ramucirumab. Kaplan-Meier curves were constructed for comparisons, and log rank tests were performed to assess for significance. Propensity matching or other attempts to reduce confounding were not performed given the small sample size of the study and risks of confusing the outcome.

\section{Results}

We identified 513 patients who met the inclusion criteria, 460 of whom received at least one dose of docetaxel without receiving ramucirumab. Of these, 41 patients had EGFR mutant disease. There were 53 patients with NSCLC who received docetaxel and ramucirumab. Of these, 18 patients had EGFR mutant disease. The median follow-up time, defined as time from initiation of therapy to patient death or end of data collection, was 7.1, 10.7 and 8.8 months for the EGFR-doce/ram, EGFR-doce and WT-doce/ram cohorts respectively. The median dates of enrollment were 11/20/2018 (IQR 5/13/2018-12/30/2019), 10/24/2015 (IQR 10/17/2012-12/31/2018) and 9/24/2018 (IQR 5/14/2016-10/28/2019) for the EGFR-doce/ram, EGFRdoce and WT-doce/ram cohorts respectively.

Patient demographics and pre-treatment variables are presented in Table 1. Patients with EGFR mutations were younger than those without and included more female patients. Performance status was not always ascertainable but showed a similar spread across cohorts with the majority of patients being ECOG 1 or 2. The EGFR-doce cohort had a higher proportion of brain metastases $(63 \%)$ prior to treatment compared to the EGFR-doce/ram (39\%) and the WT-doce/ram (37\%) cohorts. The presence of liver metastases was similar across all three cohorts. The EGFRdoce/ram (44\%) and EGFR-doce (51\%) cohorts had more frequent prior exposure to bevacizumab compared to the WT-doce/ram cohort (17\%). Among the patients with EGFR mutant disease, the EGFR-doce cohort had more frequent exposure to first generation TKIs compared to the 
Table 1 Demographics and pre-treatment variables

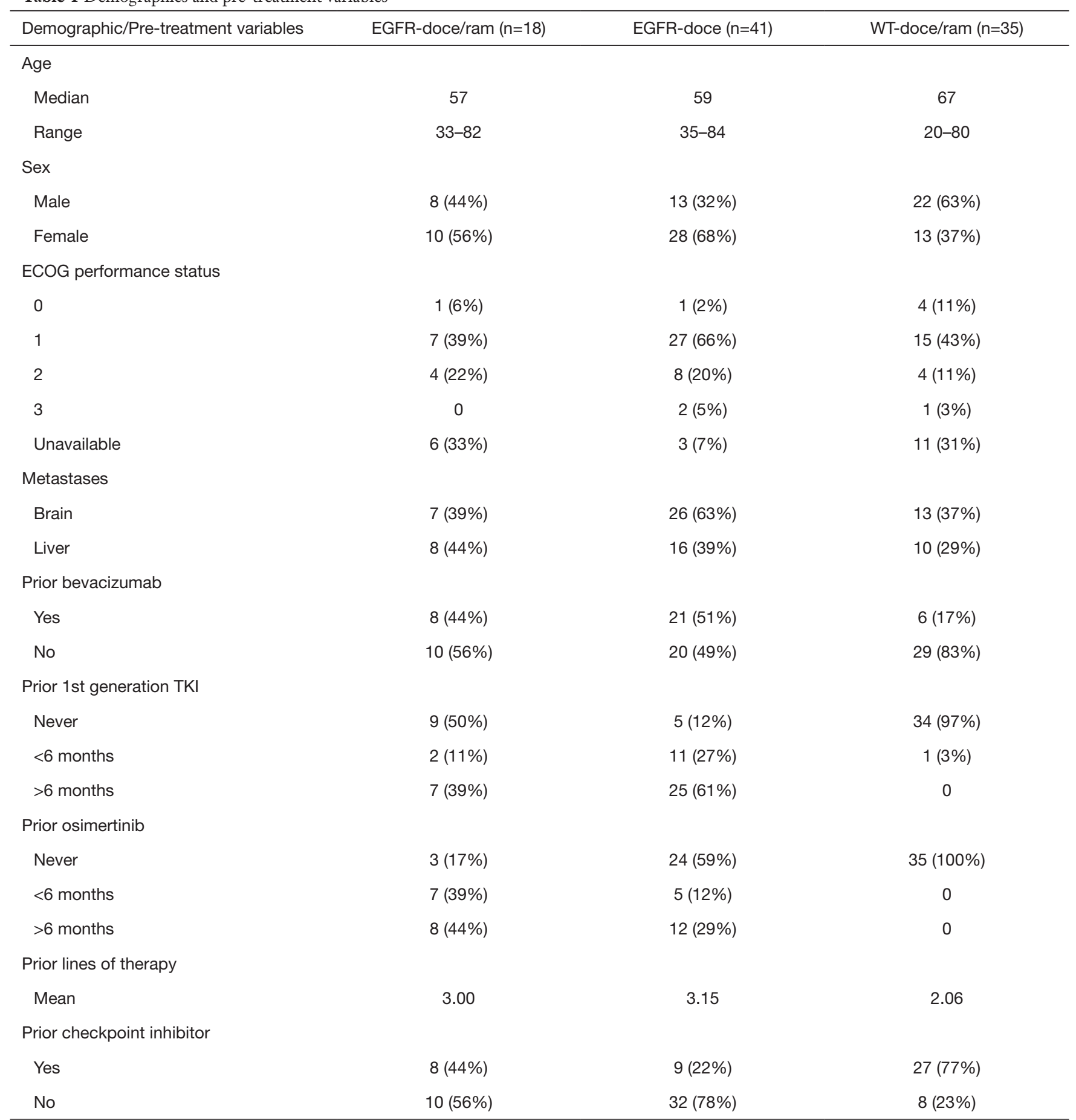

EGFR-doce, patients with EGFR mutant disease who received docetaxel only; EGFR-doce/ram, patients with EGFR mutant disease who received docetaxel and ramucirumab; WT-doce/ram, patients with wild type disease who received docetaxel and ramucirumab; EGFR, epidermal growth factor receptor; TKI, tyrosine kinase inhibitor. 
Table 2 Time on therapy

\begin{tabular}{|c|c|c|c|}
\hline Months on treatment & EGFR-doce/ram ( $n=18)$ & EGFR-doce $(n=41)$ & WT-doce/ram $(n=35)$ \\
\hline Median $(95 \% \mathrm{Cl})$ & $1.4(0.7-5.3)$ & $1.4(0.9-2.4)$ & $2.3(1.6-4.1)$ \\
\hline Censored & 0 & 1 & 4 \\
\hline \multicolumn{4}{|l|}{ Ramucirumab } \\
\hline Censored & 0 & NA & 4 \\
\hline
\end{tabular}

EGFR-doce, patients with EGFR mutant disease who received docetaxel only; EGFR-doce/ram, patients with EGFR mutant disease who received docetaxel and ramucirumab; WT-doce/ram, patients with wild type disease who received docetaxel and ramucirumab; EGFR, epidermal growth factor receptor; NA, not available.

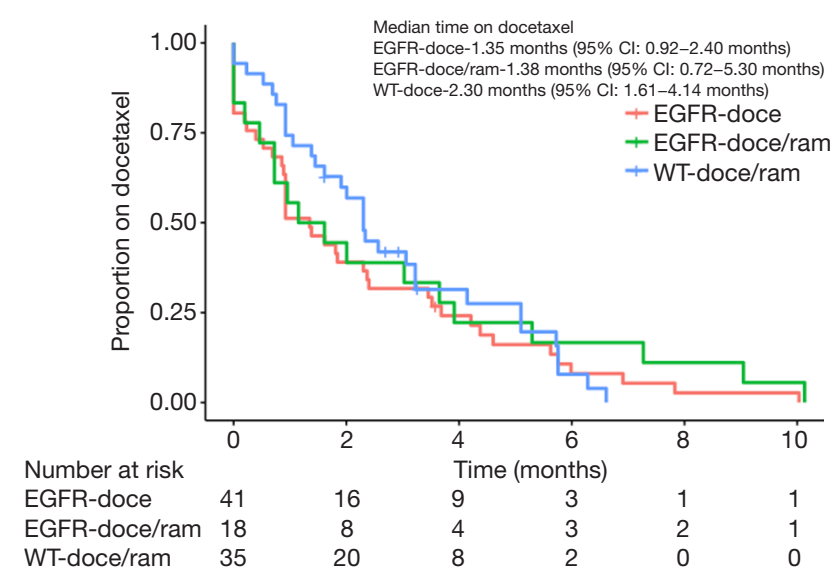

Figure 1 EGFR-doce, patients with EGFR mutant disease who received docetaxel only; EGFR-doce/ram, patients with EGFR mutant disease who received docetaxel and ramucirumab; WTdoce/ram, patients with wild type disease who received docetaxel and ramucirumab; EGFR, epidermal growth factor receptor.

EGFR-doce/ram cohort. The EGFR-doce/ram cohort had a lower proportion of patients who had never been exposed to osimertinib (17\%) compared to the EGFR-doce cohort (59\%). The WT-doce/ram cohort had fewer average lines of prior therapy and higher rates of prior immunotherapy.

The time on therapy is shown in Table 2 for both docetaxel and ramucirumab. Figures 1 and 2 show the time on therapy for all groups for docetaxel and ramucirumab. Patients in the EGFR-doce/ram cohort had a median time on docetaxel of 1.4 months (95\% CI: 0.72-5.20 months). They had a median of 0.8 months (95\% CI: 0.2-6.5 months) on ramucirumab. Patients in the EGFR-doce cohort spent a median of 1.4 months (95\% CI: 0.9-2.4 months) on docetaxel.

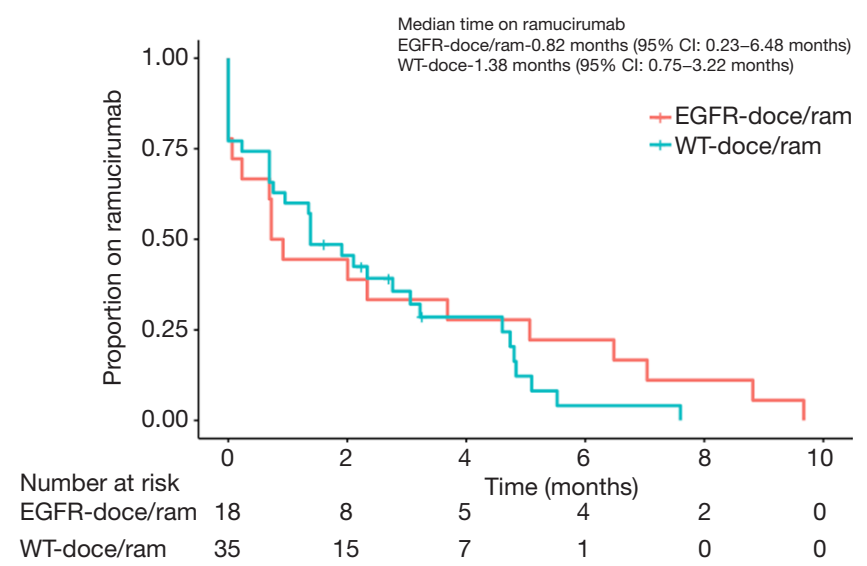

Figure 2 EGFR-doce/ram, patients with EGFR mutant disease who received docetaxel and ramucirumab; WT-doce/ram, patients with wild type disease who received docetaxel and ramucirumab; EGFR, epidermal growth factor receptor.

Patients in the WT-doce/ram cohort spent a median of 2.3 months (95\% CI: 1.6-4.1 months) on docetaxel and 1.4 months (95\% CI: 0.8-3.2 months) on ramucirumab. At the conclusion of data collection, there were four patients in the WT-doce/ram cohort and one patient in the EGFRdoce cohort still on treatment. Of the 18 patients in the EGFR-doce/ram cohort who discontinued therapy, 11 (61\%) were due to progression or death, three (17\%) being due to toxicity, and four (22\%) were unknown. Of the 31 patients in the WT-doce/ram cohort who discontinued therapy, 17 (55\%) were due to death or progression, 8 (26\%) were due to toxicity, and $6(19 \%)$ were unknown. Of the 41 patients in the EGFR-doce cohort who discontinued 


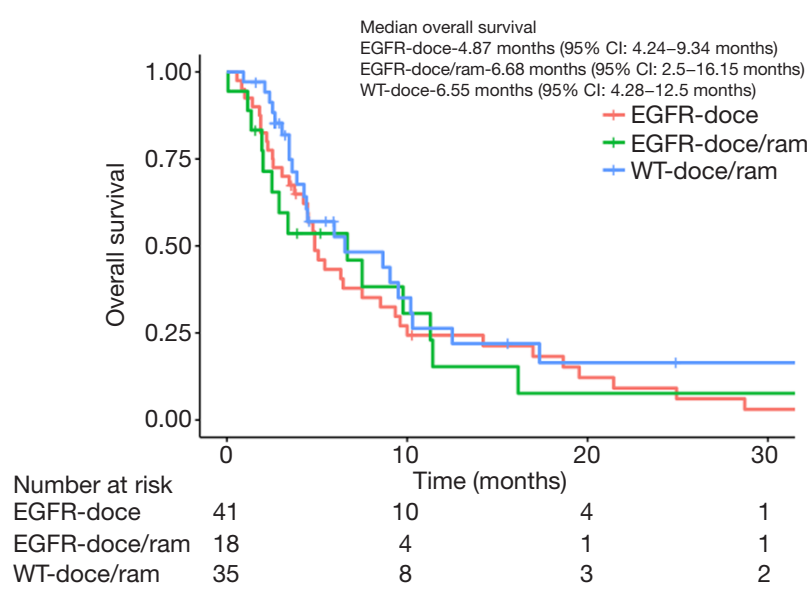

Figure 3 EGFR-doce, patients with EGFR mutant disease who received docetaxel only; EGFR-doce/ram, patients with EGFR mutant disease who received docetaxel and ramucirumab; WTdoce/ram, patients with wild type disease who received docetaxel and ramucirumab; EGFR, epidermal growth factor receptor.

therapy, $26(63 \%)$ were due to death or progression, 10 (24\%) were due to toxicity, and 5 (12\%) were unknown. There was no significant difference in time on ramucirumab between the EGFR-doce/ram and WT-doce/ram cohorts $(\mathrm{P}=0.5)$. There was also no significant difference in time on docetaxel between the EGFR-doce/ram cohort and the EGFR-doce cohort $(\mathrm{P}=0.6)$ or when comparing the EGFRdoce/ram cohort to the WT-doce/ram $(\mathrm{P}=0.9)$.

Overall survival data for the three cohorts is presented Figure 3. The EGFR-doce/ram cohort had an overall survival of 6.7 months (95\% CI: 2.5-16.2 months). The EGFRdoce cohort had an overall survival of 4.9 months (95\% CI: 4.2-12.5 months). The WT-doce/ram cohort had an overall survival of 6.6 months (95\% CI: 4.3-12.8 months). Log rank tests found no significant differences in the overall survival between the EGFR-doce/ram cohort and the EGFR-doce cohort $(\mathrm{P}=0.9)$. There was also no significant difference in overall survival between the EGFR-doce/ram cohort and the WT-doce/ram cohort $(\mathrm{P}=0.4)$.

\section{Discussion}

We conducted this retrospective cohort review with the hypothesis that patients with EGFR mutant NSCLC would have better outcomes with docetaxel and ramucirumab, as compared to wild-type patients who received docetaxel and ramucirumab or patients with EGFR mutant disease who received docetaxel alone. However, this study did not show a significant difference between time on treatment or overall survival among the three cohorts. Compared to data presented in REVEL, both EGFR-doce/ram and WT-doce/ram had shorter time on treatment than the docetaxel and ramucirumab cohort's progression free survival of 4.5 months (2). This difference is likely driven by both the difference in outcome measurement-in which time on treatment may be shorter than PFS when measured from the dates of first to last dose-as well as the unselected "real world" patient population in our study. Overall survival of all three cohorts in this study was shorter than that reported for the REVEL combination cohort (10.5 months) and the control cohort (9.1 months), likely representing the difference between clinical trial and unselected populations (2). In our study, there was a trend towards patients spending more time on docetaxel than ramucirumab among the EGFR-doce/ram and WT-doce/ ram cohorts. Additionally, visual inspection suggests that patients with wild type NSCLC performed slightly better than the other groups particularly early on, which did not support the hypothesis that this regiment would perform better in EGFR mutant NSCLC. Overall, our data do not demonstrate that EGFR mutant patients are likely to have more benefit with docetaxel and ramucirumab, and surprisingly this group did numerically worse than the wild type patients receiving the same treatment.

There were several limitations to our study. Most importantly, the initial choice of clinicians to give ramucirumab to patients is a significant counfounder that we were unable to control for. We also note that patients in the combination cohorts were on ramucirumab for shorter than docetaxel potentially diminishing the potential effect of ramucirumab. Proportionally, the reasons for discontinuation were similar among all three cohorts. We were also limited significantly by the retrospective nature, small sample size and temporal constraints of the cohorts in our study. The sample size precluded statistical adjustment for potential confounding factors, such as poor performance status, metastatic disease distribution, or prior exposure to bevacizumab or ICI therapy. There was a clear difference in the rates of prior exposure to osimertinib between EGFR mutant cohorts driven by a temporal association with later patients more likely to receive both osimertinib as well as ramucirumab. It is unclear how frontline exposure to these medications would affect response to these agents in later lines of therapy. Prior lines of therapy and type of EGFR mutation including the presence of T790M was possibly influential although not stratified in our study. We also note 
that the EGFR cohorts were most often receiving docetaxel or docetaxel with ramucirumab one line of therapy later on average than the wild type cohort which is likely explained by the first line use of EGFR inhibitors. Further, there was likely significant patient and clinician variability on decisions to initiate ramucirumab for individual patients, as well as remain on this therapy, which could have introduced systematic error based on the small pool of treating clinicians in our institution.

There remains a paucity of randomized, prospective data about the added effect of ramucirumab with docetaxel in second line settings among EGFR mutant disease. A recent retrospective study of NSCLC patients treated with the combination of docetaxel and ramucirumab demonstrated that EGFR patients have a superior PFS (5.6 vs. 3.7 months) (7). It is intriguing that this study showed a numerically improved PFS when compared to the REVEL data (2), suggesting that EGFR mutant disease is especially vulnerable to VEGF inhibition in the salvage setting. Despite our data, the incorporation of VEGF therapy in first line and salvage therapy as well as the incorporation of immunotherapy in patients with EGFR mutant NSCLC both remain active areas of investigation. Recently, a phase I study of the addition of ramucirumab to osimertinib in EGFR mutant disease progressing after first generation TKI with the T790M mutation showed encouraging results with a median PFS of 11 months (8). A very recent phase II study of the addition of bevacizumab to osimertinib in EGFR mutant NSCLC with progression on first generation TKI, showed no significant improvement in PFS or OS with the addition of bevacizumab (9). Currently, a phase II study comparing osimertinib with and without ramucirumab as frontline treatment for EGFR mutant NSCLC is actively enrolling (10). Active investigation into VEGF inhibitor and ICI combinations currently include a phase II study examining the combination of docetaxel, ramucirumab and pembrolizumab in patients who have progressed through platinum based therapy with or without immunotherapy, as well as a phase II trial comparing ramucirumab and pembrolizumab with standard of care therapy in the same patient population $(11,12)$.

Our study offers real-world estimates to clinicians and patients about the length of time they can expect to receive benefit from docetaxel and ramucirumab. With regard to patients with EGFR mutant NSCLC, the authors recommend continued consideration of ramucirumab when clinically indicated, but a remaining question is whether to anticipate better performance than docetaxel alone. In the future, we support further research into the backbone of osimertinib with ramucirumab, which may improve outcomes in the first line setting for these patients. We eagerly anticipate the results from the II study comparing osimertinib with or without ramucirumab in EGFR mutant, TKI naïve NSCLC and a similar study with osimertinib and bevacizumab (10). Furthermore, the ongoing pivotal phase III study of osimertinib with or without bevacizumab as first line therapy for EGFR mutant NSCLC has the potential to change the standard of care first line therapy in this disease (13).

\section{Acknowledgments}

We acknowledge STARR, our institutional outcomes database and the support required to maintain it from the Stanford University School of Medicine.

Funding: None.

\section{Footnote}

Reporting Checklist: The authors have completed the STROBE reporting checklist. Available at https://dx.doi. org/10.21037/jtd-21-557

Data Sharing Statement: Available at https://dx.doi. org/10.21037/jtd-21-557

Conflicts of Interest: Both authors have completed the ICMJE uniform disclosure form (available at https:// dx.doi.org/10.21037/jtd-21-557). JWN reports grants and personal fees from Takeda during the conduct of the study; personal fees from AstraZeneca, grants, personal fees, and non-financial support from Genentech/Roche, grants, personal fees, and non-financial support from Exelixis, personal fees from Jounce Therapeutics, personal fees from Eli Lilly and Company, personal fees from Calithera Biosciences, personal fees from Amgen, personal fees from Lovance Biotherapeutics, personal fees from Blueprint Pharmaceuticals, personal fees from Regeneron Pharmaceuticals, personal fees from Natera, grants and non-financial support from Merck, grants and non-financial support from Novartis, grants and non-financial support from Boehringer Ingelheim, grants and non-financial support from Nektar Therapeutics, grants and non-financial support from Adaptimmune, grants and non-financial support from GSK, grants and non-financial support from Janssen, and grants and non-financial support from AbbVie 
outside the submitted work. TEC has no conflicts to disclose.

Ethical Statement: The authors are accountable for all aspects of the work in ensuring that questions related to the accuracy or integrity of any part of the work are appropriately investigated and resolved. The study was conducted in accordance with the Declaration of Helsinki (as revised in 2013). The study was approved by institutional ethics board of Stanford University (IRB \#21319) and individual consent for this retrospective analysis was waived.

Open Access Statement: This is an Open Access article distributed in accordance with the Creative Commons Attribution-NonCommercial-NoDerivs 4.0 International License (CC BY-NC-ND 4.0), which permits the noncommercial replication and distribution of the article with the strict proviso that no changes or edits are made and the original work is properly cited (including links to both the formal publication through the relevant DOI and the license). See: https://creativecommons.org/licenses/by-nc-nd/4.0/.

\section{References}

1. Spratlin JL, Cohen RB, Eadens M, et al. Phase I pharmacologic and biologic study of ramucirumab (IMC1121B), a fully human immunoglobulin G1 monoclonal antibody targeting the vascular endothelial growth factor receptor-2. J Clin Oncol 2010;28:780-7.

2. Garon EB, Ciuleanu TE, Arrieta O, et al. Ramucirumab plus docetaxel versus placebo plus docetaxel for secondline treatment of stage IV non-small-cell lung cancer after disease progression on platinum-based therapy (REVEL): a multicentre, double-blind, randomised phase 3 trial. Lancet 2014;384:665-73.

3. Nilsson MB, Robichaux J, Herynk MH, et al. Altered Regulation of HIF- $1 \alpha$ in Naive- and Drug-Resistant EGFR-Mutant NSCLC: Implications for a Vascular Endothelial Growth Factor-Dependent Phenotype. J Thorac Oncol 2021;16:439-51.

Cite this article as: Ellis-Caleo T, Neal JW. The role of ramucirumab with docetaxel in epidermal growth factor receptor mutant and wild-type non-small cell lung cancer. J Thorac Dis 2021;13(8):4864-4871. doi: 10.21037/jtd-21-557
4. Nakagawa K, Garon EB, Seto T, et al. Ramucirumab plus erlotinib in patients with untreated, EGFRmutated, advanced non-small-cell lung cancer (RELAY): a randomised, double-blind, placebo-controlled, phase 3 trial. Lancet Oncol 2019;20:1655-69.

5. Saito H, Fukuhara T, Furuya N, et al. Erlotinib plus bevacizumab versus erlotinib alone in patients with EGFR-positive advanced non-squamous non-small-cell lung cancer (NEJ026): interim analysis of an open-label, randomised, multicentre, phase 3 trial. Lancet Oncol 2019;20:625-35.

6. Yoh K, Hosomi Y, Kasahara K, et al. A randomized, double-blind, phase II study of ramucirumab plus docetaxel vs placebo plus docetaxel in Japanese patients with stage IV non-small cell lung cancer after disease progression on platinum-based therapy. Lung Cancer 2016;99:186-93.

7. Furuya N, Ito K, Sakaguchi T, et al. The Impact of EGFR Mutation Status and Brain Metastasis for Non-Small Cell Lung Cancer Treated with Ramucirumab plus Docetaxel. Oncology 2020;98:661-8.

8. Yu HA, Paz-Ares LG, Yang JC, et al. Phase I Study of the Efficacy and Safety of Ramucirumab in Combination with Osimertinib in Advanced T790M-positive EGFRmutant Non-small Cell Lung Cancer. Clin Cancer Res 2021;27:992-1002.

9. Akamatsu H, Toi Y, Hayashi H, et al. Efficacy of Osimertinib Plus Bevacizumab vs Osimertinib in Patients With EGFR T790M-Mutated Non-Small Cell Lung Cancer Previously Treated With Epidermal Growth Factor Receptor-Tyrosine Kinase Inhibitor: West Japan Oncology Group 8715L Phase 2 Randomized Clinical Trial. JAMA Oncol 2021;7:386-94.

10. Available online: https://clinicaltrials.gov/ct2/show/NCT0 3909334? term $=03909334 \&$ draw $=2 \&$ rank $=1$

11. Available online: https://clinicaltrials.gov/ct2/show/NCT0 4340882 ? term $=04340882 \&$ draw $=2$ \& rank $=1$

12. Available online: https://clinicaltrials.gov/ct2/show/NCT0 3971474? term $=03971474 \&$ draw $=2 \&$ rank $=1$

13. Available online: https://clinicaltrials.gov/ct2/show/NCT0 4181060? term $=04181060 \&$ draw $=2 \&$ rank $=1$ 